\title{
Influence of Electric Field on AC Magnetic Susceptibility of a Mineral Oil Based Ferrofluid
}

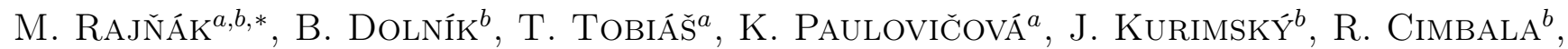 \\ P. KOPČANSKÝ ${ }^{a}$ AND M. TIMKO ${ }^{a}$ \\ ${ }^{a}$ Institute of Experimental Physics, Slovak Academy of Sciences, Watsonova 47, 040 01, Košice, Slovakia \\ ${ }^{b}$ Faculty of Electrical Engineering and Informatics, Technical University of Košice, Letná 9, 04200 Košice, Slovakia
}

\begin{abstract}
In a ferrofluid, the magnetic susceptibility at low magnetic fields is sensitive to the size and shape of magnetic nanoparticles and their concentration in a liquid carrier. In this paper we report on the AC magnetic susceptibility of a ferrofluid based on a mineral oil and iron oxide nanoparticles coated with oleic acid. As this type of ferrofluid is of increasing interest for electrical engineering applications, we investigate the effect of an external electric field and an electric current flowing through the ferrofluid on the AC magnetic susceptibility at ambient conditions. It is known that the action of the electric field on the oil based ferrofluids can result in the particle assembling. Then, the induced structural changes may affect the magnetic susceptibility of the bulk ferrofluid. In our experimental study we observed that the ferrofluid magnetic susceptibility decreases with increasing electric field. However, a heating effect with increasing electric field was observed too. Hence, it is concluded that besides the structural changes, the Joule heating has an obvious impact on the magnetic susceptibility of the ferrofluid in the external electric field.
\end{abstract}

DOI: 10.12693/APhysPolA.133.567

PACS/topics: 47.65.Cb, 75.30.Cr, 47.65.-d

\section{Introduction}

AC magnetic susceptibility measurements are recognized as an important tool in the characterization of magnetic nanoparticle systems which exhibit superparamagnetism [1]. In stable colloidal suspensions of magnetic nanoparticles in a liquid carrier (ferrofluids), there are two distinct mechanisms by which the magnetization may relax after an $\mathrm{AC}$ magnetic field has been applied to a ferrofluid. The first one is the rotational Brownian motion of the particle within the carrier liquid, with its magnetic moment blocked in an axis of easy magnetization. The other one is represented by the rotation of the magnetic moment within the particle (Néel relaxation) $[2,3]$. Both, Brownian and Néel relaxation mechanisms, have been often identified and investigated by measurements of frequency dependent complex magnetic susceptibility $\chi(\omega)$, which is vital for an understanding of the dynamic behavior of ferrofluids [4-6]. The superparamagnetic nanoparticles in a ferrofluid are often considered as non-interacting particles, especially for a low concentration case. On the other hand, for concentrated ferrofluids, the dipole-dipole interactions are predicted to increase the Brownian relaxation times, while the Néel mechanism is not affected significantly. Such effects have been detected experimentally [7] and recently novel numerical and theoretical approaches were published $[8,9]$. By means of temperature and concentration dependent AC magnetic susceptibility investigation and theoretical

*corresponding author; e-mail: rajnak@saske.sk analysis it was shown [10] how to separate the effects of superparamagnetic polydispersity and interparticle magnetic interactions on the dynamic spectra, which deepens the understanding of the dynamic nanoparticle selfassembly.

The ferrofluid nanoparticle assembly formation has been intensively studied in external magnetic fields by various experimental methods $[11,12]$. It is clear that the structural changes in the ferrofluid have a significant impact on the ferrofluid's dynamic magnetic response. Recently, it was shown that similar assemblies can be induced by electric fields. Especially ferrofluids based on insulating liquids are characterized by a high dielectric contrast between the particles and the base liquid, which results in the effective particle polarization and dielectrophoresis leading to the particle assembling $[13,14]$. On the other hand, this type of ferrofluids is of increasing interest due to their potential application in high voltage engineering [15].

Following the observed ferrofluid structural changes induced by electric fields and the potential application of the insulating liquid based ferrofluids in high voltage engineering, it is reasonable to verify the effect of the electric field on the magnetic response too. In this paper we have experimentally investigated the AC magnetic susceptibility of a ferrofluid based on a mineral oil under an electric field of various intensities.

\section{Experimental}

For the intended experimental study we have prepared a ferrofluid based on an insulating mineral oil with permittivity of 1.9 and density of $0.821 \mathrm{~g} / \mathrm{cm}^{3}$. Iron oxide nanoparticles with an average diameter of $10 \mathrm{~nm}$ were 
synthesized by a chemical co-precipitation and coated with oleic acid in the well proven way [16]. The magnetic volume fraction of the investigated sample was $4.7 \%$, as determined from magnetic measurements (Vibrating Sample Magnetometer, Cryogenic Limited), corresponding to the magnetization of saturation of $20.8 \mathrm{kA} / \mathrm{m}$. Temperature dependent magnetization measurements in ZFC (Zero Field Cooled) and FC (Field Cooled) regime revealed the typical splitting of the ZCF and FC curves for superparamagnetic systems (Fig. 1). The broad shoulder around $50 \mathrm{~K}$ on the ZFC curve reflects the distribution of the blocking temperatures associated with the particle size distribution. The maximum at $220 \mathrm{~K}$ is related to the pour point of the oil.

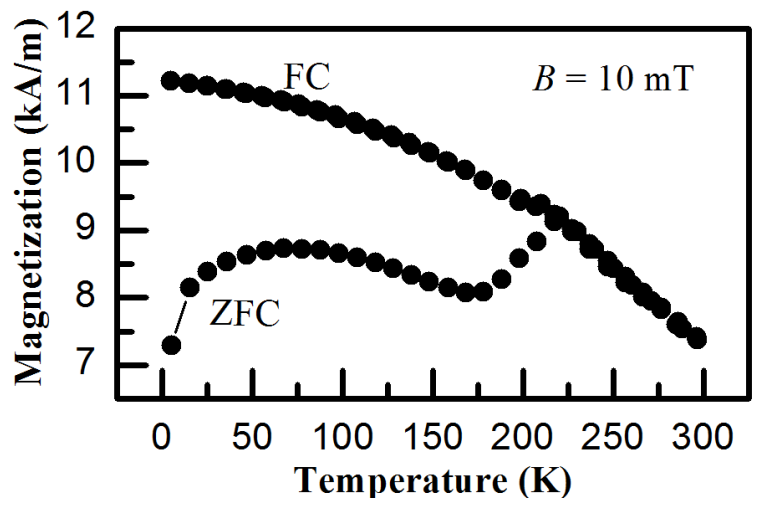

Fig. 1. Magnetization curve after zero field cooling (ZFC) and field cooling (FC) measured at a constant magnetic field of $10 \mathrm{mT}$.

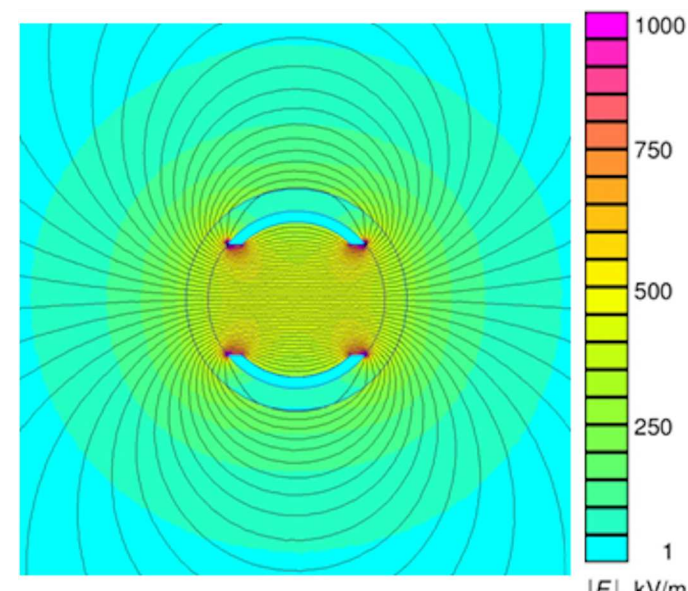

Fig. 2. Simulated equipotential field lines and the field intensity distribution (color scale) in the cross-section of the air filled vial with the potential difference of $2.1 \mathrm{kV}$ acting on the electrodes.

The AC magnetic susceptibility was measured by an AC susceptometer DynoMag Imego with embodied thermometer near a sample holder. The excitation field amplitude was $0.5 \mathrm{mT}$. The sample $(200 \mu \mathrm{l})$ was measured in a glass vial equipped with two U-shaped brass electrodes connected to a DC (direct current) power supply. The electric field distribution in the vial cross-section was simulated by Finite Element Method Magnetics (FEMM 4.2) using the triangular element mesh and the iterative conjugate gradient solver (Fig. 2). The DC electric current flowing through the sample was measured by a multimeter. The presented current values were taken approximately 1 minute after the voltage has been applied.

\section{Results and discussion}

The measured real magnetic susceptibility spectra of the studied ferrofluid under various electric field conditions are presented in Fig. 3. The presented values correspond purely to the ferrofluid susceptibility, as the vial and electrodes contributions (background) have been subtracted. In the absence of the electric field $(0 \mathrm{kV})$, the depicted spectrum represents a typical profile corresponding to a polydispersed nanoparticle system [3]. Thus, the moderately decreasing susceptibility with increasing frequency implies the distribution of relaxation times with both mechanisms contributing to the magnetization. This is qualitatively in agreement with the ZFC shoulder and the related particle size distribution.

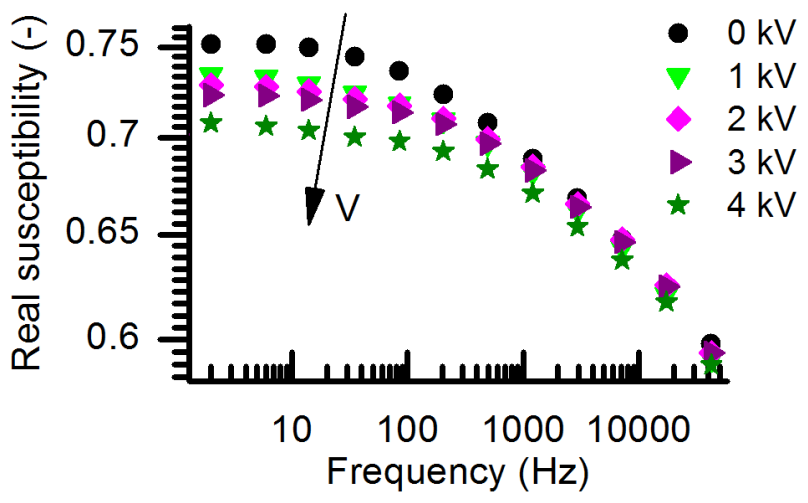

Fig. 3. Real magnetic susceptibility spectra measured on the ferrofluid exposed to various electric potential differences.

It can be seen that the applied electric field does not change the profile of the susceptibility spectrum markedly. Therefore, the effective relaxation time distribution was not affected by the electric field. However, as the graph shows, the amplitude of the real susceptibility decreases with increasing electric field. This behaviour can stem from two effects. First, in accordance with the proven magnetic particle aggregation in the electric field [13], the magnetic moment of the resulting cluster may be reduced or even compensated. This can happen due to possible magnetic interactions in the formed clusters, which minimize their total energy. Thus, one could assume that the particle magnetic moments interact in the clusters in a way leading to the compensated total magnetic moment of the cluster. Subsequently, only the 
individual particles would respond the applied magnetic field and the ferrofluid exhibits the lower magnetic susceptibility.

The other accountable reason for the measured decrease in the real susceptibility amplitude is associated with the heating effect of the ferrofluid due to the passing electric current through it. Even though the ferrofluid is based on the insulating oil, it contains a certain amount of charge carriers represented predominantly by water (moisture) and ionic impurities from residual surfactant and unreacted unwashed ions. Thus, when the potential difference is applied to the electrodes, the charge carriers are accelerated and collide with the other ferrofluid constituents. This Joule heating then increases the ferrofluid temperature, and as it is well known, the heating power is proportional to the product of the ferrofluid resistance and the square of the current. The heating effect of the sample is proven in Fig. 4, showing the increase in the electric current with increasing voltage and the related increase in temperature of the measured ferrofluid. Then, following the Curie-Weiss law, the magnetic susceptibility must decrease. This is again in accordance with the ZFC-FC curve (Fig. 1).

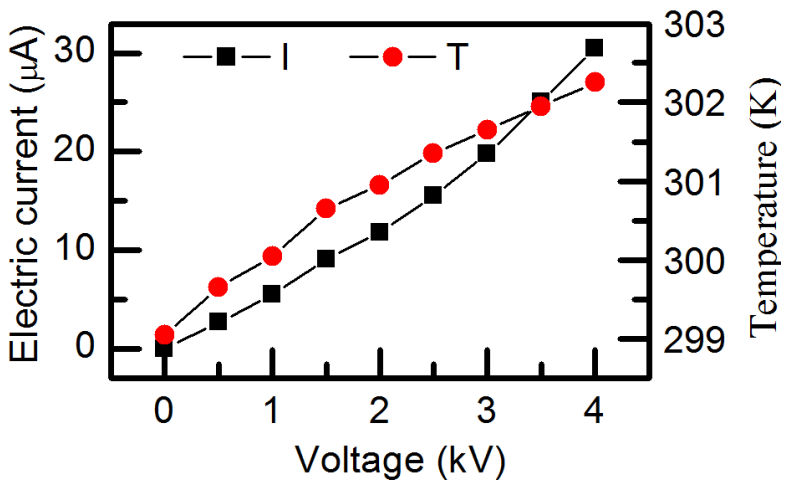

Fig. 4. Average ferrofluid temperature $\mathrm{T}$ and electric current I flowing through the ferrofluid during the AC magnetic susceptibility measurements with various potential differences (voltage) on the electrodes.

\section{Conclusions}

The presented study demonstrates the AC magnetic susceptibility of a ferrofluid based on the insulating mineral oil under the DC electric field. It was shown that with the increasing electric voltage (and resulting electric field) acting on the ferrofluid, the magnetic susceptibility spectrum decreases in amplitude but does not change in the profile. The electric field driven particle assembly and subsequent magnetic moment interactions in the formed assemblies may account for the observed susceptibility drop. However, as clearly shown in this study, the Joule heating of the sample is more probable and intuitive reason.

\section{Acknowledgments}

This work was supported by the Slovak Academy of Sciences and Ministry of Education in the framework of projects VEGA Nos. 2/0141/16, 2/0016/17 and 1/0340/18, Ministry of Education Agency for structural funds of EU Project ITMS 26220220061, COST CA15119NANOUPTAKE, and Slovak Research and Development Agency under the Contract No. APVV-150438 and APVV-15-0453 (M-Vision).

\section{References}

[1] D. Serantes, D. Baldomir, Open Surf. Sci. J. 4, 71 (2012).

[2] A.P. Guimarăes, Principles of Nanomagnetism, Springer Science \& Business Media, Berlin Heidelberg 2009.

[3] P. Fannin, J. Magn. Magn. Mater. 252, 59 (2002).

[4] J. Zhang, C. Boyd, W. Luo, Phys. Rev. Lett. 77, 390 (1996).

[5] I. Mălăescu, N. Ştefu, L. Gabor, J. Magn. Magn. Mater. 234, 299 (2001).

[6] P.C. Fannin, B.K.P. Scaife, S.W. Charles, J. Magn. Magn. Mater. 85, 54 (1990).

[7] G.T. Landi, Phys. Rev. B 89, 14403 (2014).

[8] A.O. Ivanov, V.S. Zverev, S.S. Kantorovich, Soft Matter 12, 3507 (2016).

[9] J. O. Sindt, P.J. Camp, S.S. Kantorovich, E.A. Elfimova, A.O. Ivanov, Phys. Rev. E 93, 63117 (2016).

[10] A.O. Ivanov, S.S. Kantorovich, V.S. Zverev, E.A. Elfimova, A.V. Lebedev, A.F. Pshenichnikov, Phys. Chem. Chem. Phys. 18, 18342 (2016).

[11] D. Heinrich, A.R. Goni, A. Smessaert, S.H.L. Klapp, L.M.C. Cerioni, T.M. Osan, D.J. Pusiol, C. Thomsen, Phys. Rev. Lett. 106, 208301, (2011).

[12] J. Kúdelčík, P. Bury, Š. Hardoň, M. Sedlačík, M. Mrlík, in: 2016 ELEKTRO Strbske Pleso, IEEE, 2016 p. 624 .

[13] M. Rajnak, V.I. Petrenko, M.V. Avdeev, O.I. Ivankov, A. Feoktystov, B. Dolnik, J. Kurimsky, P. Kopcansky, M. Timko, Appl. Phys. Lett. 107, 73108, (2015).

[14] M. Rajnak, J. Kurimsky, B. Dolnik, P. Kopcansky, N. Tomasovicova, E. A. Tacuescu-Moaca. M. Timko, Phys. Rev. E 90, 32310, (2014).

[15] M. Rafiq, Y. Lv, C. Li, M. Rafiq, Y. Lv, C. Li, J. Nanomater. 2016, e8371560, (2016).

[16] L. Vékás, D. Bica, M.V. Avdeev, China particuology 5, 1 (2007) 